\title{
The Child-Turcotte Classification: From Gestalt to Sophisticated Statistics and Back
}

\author{
Guadalupe Garcia-Tsao ${ }^{1,2}$
}

Published online: 30 September 2016

(C) Springer Science+Business Media New York (Outside the USA) 2016

In a landmark article published 52 years ago, Child and Turcotte, two surgeons who performed portacaval shunt surgery, classified patients with cirrhosis in three "functional hepatic reserve" categories: A, B, and C, corresponding to minimally, moderately, and severely altered hepatic functional reserve, respectively [1]. This classification was based on five variables, three clinical (ascites, encephalopathy, and nutritional status), and two biochemical (serum albumin and bilirubin). Notably, the selection of these variables was based on clinical experience and not on multivariable analysis, which at the time was not widely used, although they did perform actuarial survival analysis to demonstrate statistically significant differences for the probabilities of 3- and 10-year survival among the three groups.

The Child-Turcotte classification was modified by Pugh et al. [2] by substituting the normalized ratio (INR) for nutritional status and by arbitrarily adjusting limits for serum albumin and better defining the encephalopathy grading system (Fig. 1). Pugh et al. also assigned 1, 2, or 3 points to each of the five variables enabling the calculation of a score, the Child-Turcotte-Pugh (CTP) score, ranging from 5 to 15 , with scores of 5 and 6 corresponding to CTP class A, scores of 7-9 corresponding to CTP class B, and scores of 10-15 corresponding to CTP class C (Fig. 1).

Guadalupe Garcia-Tsao

guadalupe.garcia-tsao@yale.edu

1 Section of Digestive Diseases, Yale University School of Medicine, 333 Cedar St - 1080 LMP, New Haven, CT 06510, USA

2 Section of Digestive Diseases, VA-CT Healthcare System, West Haven, CT, USA
Many years later, Malinchoc et al. [3] defined a numerical prognostic score for patients with cirrhosis also subjected to portosystemic shunt (shunts in the subjects that they analyzed were placed via the transjugular route rather than surgically), which included three objective laboratory variables (bilirubin, INR, and creatinine) selected by multivariable analysis. This model for end-stage liver disease (MELD) score has been particularly useful in predicting death in patients with decompensated cirrhosis, likely because the transjugular intrahepatic portosystemic shunt (TIPS) was performed in patients with refractory ascites or recurrent variceal hemorrhage and is therefore used in liver transplant allocation [4]. Since in the times of Child and Turcotte, surgical shunts were performed even in compensated patients, before data were available demonstrating significantly higher encephalopathy rates and a tendency for a higher mortality in shunted patients [5], its prognostic value spans all stages of cirrhosis.

The study by Kaplan et al. [6] in this issue of Digestive Diseases and Sciences represents the largest validation study of the original CTP class in a thoughtful analysis of a large cohort of US veterans obtained from an administrative database. Based on sophisticated statistics, the investigators confirm that CTP A versus B versus $\mathrm{C}$ is associated with a progressively poorer prognosis, informing their proposal of new evidence-based limits for the laboratory parameters (albumin, bilirubin, and INR) that are more precise than the original limits in their prediction of death than the original CTP score.

While the scientific community should strive for precision, fluctuations in these tests due to concurrent events (e.g., dehydration, hemolysis, and sepsis) or to current therapies such as the increasing use of intravenous albumin, render these limits inherently imprecise. Therefore, whether serum albumin levels should be assigned two CTP 
Fig. 1 Original table as published in the paper by Pugh et al. [2]

\section{Table I.-Grading of Severity of Liver Disease}

\begin{tabular}{|c|c|c|c|}
\hline \multirow{2}{*}{$\begin{array}{c}\text { CliniCAL AND BIOCHEMICAL } \\
\text { MEASUREMENTS }\end{array}$} & \multicolumn{3}{|c|}{$\begin{array}{c}\text { POINTS SCORED FOR INCREASING } \\
\text { ABNORMALITY }\end{array}$} \\
\hline & $\mathbf{I}$ & 2 & 3 \\
\hline $\begin{array}{l}\text { Encephalopathy (grade) } \\
\text { Ascites } \\
\text { Bilirubin (mg. per } 100 \mathrm{ml} \text { ). } \\
\text { Albumin (g. per 100 ml.) } \\
\text { Prothrombin time (sec. prolonged) } \\
\text { For primary biliary cirrhosis:- } \\
\text { Bilirubin (mg. per I00 ml.) }\end{array}$ & $\begin{array}{c}\text { None } \\
\text { Absent } \\
I-2 \\
3 \cdot 5 \\
I-4 \\
1-4\end{array}$ & $\begin{array}{c}1 \text { and } 2 \\
\text { Slight } \\
2-3 \\
2 \cdot 8-3 \cdot 5 \\
4-6 \\
4-10\end{array}$ & $\begin{array}{c}3 \text { and } 4 \\
\text { Moderate } \\
>3 \\
<2 \cdot 8 \\
>6 \\
>10\end{array}$ \\
\hline
\end{tabular}

* According to grading of Trey, Burns, and Saunders (1966).

points if it is between 2.8 and $3.5 \mathrm{mg} / \mathrm{dL}$ (original score) or if it is between 3.3 and $3.6 \mathrm{mg} / \mathrm{dL}$ (modified score), while statistically significant, is probably of little clinical significance. Currently, when important clinical trials have stratified patients by CTP class using the original laboratory test limits, their modification could lead to confusion in the field.

More importantly, Kaplan et al. [6,7] in this and a previous study have made great strides in defining the two CTP clinical variables, ascites and encephalopathy, aspects of the CTP classification that have been considered its major shortcoming because they are subjective and are confusing not only in the stratification of patients in clinical trials but even more challenging when analyzing administrative databases.

In the original CTP classification, although ascites and hepatic encephalopathy (HE) were assessed in a crosssectional manner (Fig. 1), a patient may not have had ascites at the time of assessment due to response to diuretics or to interventions such as large-volume paracenteses or TIPS that may have been performed for refractory ascites. Therefore, the strategy adopted by Kaplan et al. [6,7] was to take a longitudinal view of these clinical variables so as to faithfully represent the clinical staging of cirrhosis with well-defined prognostic implications [8]. For ascites, a CTP score of 3 is assigned to patients with refractory ascites requiring frequent largevolume paracentesis or TIPS as well as patients with a history of spontaneous bacterial peritonitis; a score of 2 is assigned to patients requiring diuretic therapy for control of ascites but without any of the previously mentioned complications. Moreover, a CTP score of 1 is assigned to patients without clinically overt ascites (no ascites or ascites only detectable by ultrasonography [9]). For HE, a CTP score of 3 is assigned to patients with more than 1 hospitalization for HE in 6 months, while a score of 2 is assigned to patients requiring sustained lactulose and/or rifaximin for control of HE without hospitalizations for HE. To add to this, a score of 1 is assigned to patients with covert encephalopathy (no, minimal, or grade I encephalopathy [10]). When an accurate history is not available, a cross-sectional view should at least consider the use (or not) of specific therapies.

Not surprisingly, the study by Kaplan et al. [6] reports that the CTP score (whether it was the original or the modified score) is better than the "objective" MELD score in predicting mortality. MELD only discriminated 1- to 5-year outcomes accurately in patients with a CTP score $>11$, that is, in patients with decompensated cirrhosis, validating results obtained in a prospective cohort [11]. It is likely that the differences were mostly due to the correct assessment of the subjective clinical variables. In this sense, the major achievement by Kaplan et al. is the ability to correctly classify patients with cirrhosis included in an administrative database into three distinct prognostic categories, particularly when many important outcomes research and health system-based quality management strategies are currently based on these databases.

Beyond research, there is a clinical need to provide accurate prognostic information to patients with liver disease. The Child-Turcotte classification is a clinical classification conceived by astute clinicians that even now can be bested by a gestalt assessment (looking at the whole patient) rather than by assessing and adding up its five components. In fact, now that issues of malnutrition and sarcopenia have resurfaced as important prognostic factors in patients with cirrhosis [12], re-incorporation of one of the original clinical variables, nutritional status into the classification, as originally proposed over 5 decades ago by Drs. Child and Turcotte, should be reconsidered, a modification that would bring the CTP "full circle" back to its roots. 
Acknowledgments Support for this editorial was provided by the Yale Liver Center NIH P30 DK34989.

\section{References}

1. Child CG, Turcotte JG. Surgery and portal hypertension. Major Probl Clin Surg. 1964;1:1-85.

2. Pugh RN, Murray-Lyon IM, Dawson JL, Pietrini MC, Williams R. Transection of the esophagus for bleeding oesophageal varices. Br J Surg. 1973;60:646-649.

3. Malinchoc M, Kamath PS, Gordon FD, Peine CJ, Rank J, TerBorg PCJ. A model to predict poor survival in patients undergoing transjugular intrahepatic portosystemic shunts. Hepatology. 2000;31:864-871.

4. Kamath PS, Wiesner RH, Malinchoc M, et al. A model to predict survival in patients with end-stage liver disease. Hepatology. 2001;33:464-470.

5. D'Amico G, Pagliaro L, Bosch J. The treatment of portal hypertension: a meta-analytic review. Hepatology. 1995;22: 332-354.

6. Kaplan DE, Dai F, Skanderson M, et al. Recalibrating the ChildTurcotte-pugh Score to improve prediction of transplant-free survival in patients with cirrhosis. Dig Dis Sci. (Epub ahead of print). doi:10.1007/s10620-016-4239-6.

7. Kaplan DE, Dai F, Aytaman A, et al. Development and performance of an algorithm to estimate the Child-Turcotte-Pugh Score from a national electronic healthcare database. Clin Gastroenterol Hepatol. 2015;13:2333-2341.

8. Garcia-Tsao G. Natural history of cirrhosis. In: Cardenas A, Keaveny A, eds. Complications of Cirrhosis. New York: Springer; 2015.

9. Zipprich A, Seufferlein T, Dollinger MM. Subclinical ascites defines an intermediate stage between compensated and decompensated cirrhosis. Z Gastroenterol. 2012;50:996-1001.

10. Vilstrup H, Amodio P, Bajaj J, et al. Hepatic encephalopathy in chronic liver disease: 2014 Practice Guideline by the American Association for the Study of Liver Diseases and the European Association for the Study of the Liver. Hepatology. 2014;60: 715-735.

11. Ripoll C, Bari K, Garcia-Tsao G. Serum albumin can identify patients with compensated cirrhosis with a good prognosis. J Clin Gastroenterol. 2015;49:613-619.

12. Montano-Loza AJ, Meza-Junco J, Prado CM, et al. Muscle wasting is associated with mortality in patients with cirrhosis. Clin Gastroenterol Hepatol. 2012;10:166-173. 